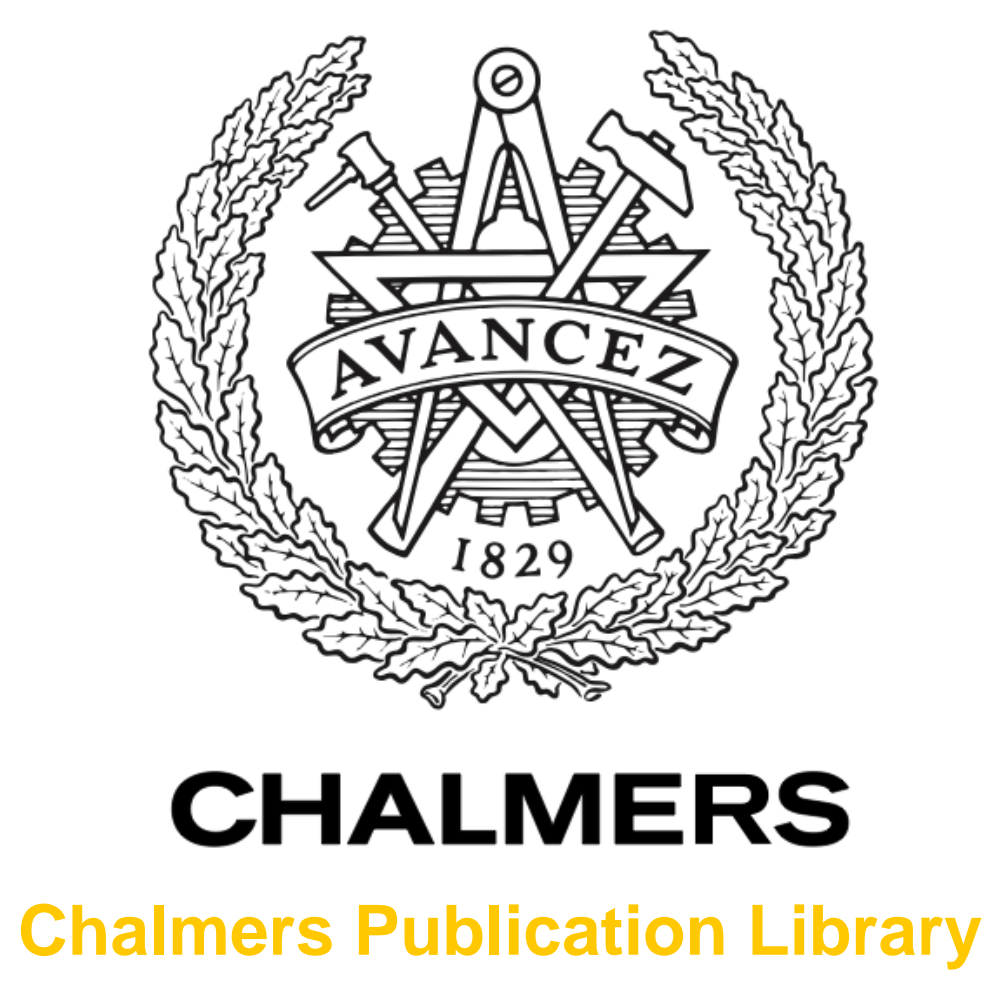

Exploiting statistical and outdated CSI in multiuser downlink transmission

This document has been downloaded from Chalmers Publication Library (CPL). It is the author's version of a work that was accepted for publication in:

International Conference on Wireless Communications and Signal Processing, WCSP 2012; Huangshan; China; 25 October 2012 through 27 October 2012

Citation for the published paper:

Jin, S. ; Wang, J. ; Matthaiou, M. (2012) "Exploiting statistical and outdated CSI in multiuser downlink transmission". International Conference on Wireless Communications and Signal Processing, WCSP 2012; Huangshan; China; 25 October 2012 through 27 October 2012

http://dx.doi.org/10.1109/WCSP.2012.6542895

Downloaded from: http://publications.lib.chalmers.se/publication/181139

Notice: Changes introduced as a result of publishing processes such as copy-editing and formatting may not be reflected in this document. For a definitive version of this work, please refer to the published source. Please note that access to the published version might require a subscription. 


\title{
Exploiting Statistical and Outdated CSI in Multiuser Downlink Transmission
}

\author{
Shi $\operatorname{Jin}^{\S}$, Jue Wang ${ }^{\S}$, Michail Matthaiou ${ }^{\ddagger}$, and Xiqi Gao ${ }^{\S}$ \\ $\S$ National Mobile Communications Research Laboratory, Southeast University \\ Nanjing 210096, P. R. China, Email: \{jinshi, wangjue, xqgao\}@ seu.edu.cn \\ ${ }^{\ddagger}$ Department of Signals and Systems, Chalmers University of Technology \\ Gothenburg SE-412 96, Sweden, Email: michail.matthaiou@chalmers.se
}

\begin{abstract}
In this paper, we propose a multiuser downlink transmission scheme, which exploits both the statistical and outdated channel state information (CSI) at the transmitter. The proposed scheme extends the outdated CSI-aided scheme, first introduced by Maddah-Ali and Tse in [1] (denoted as MAT), by sending symbols demanded by a user along its statistical eigenmodes, instead of directly sending the symbols through separate antennas, and we refer to it as statistical eigenmode-MAT (SE-MAT). Considering zero-forcing receiver, we explicitly prove that the proposed SE-MAT scheme can achieve a higher ergodic sum-rate compared to the original MAT scheme, under different correlation conditions. Moreover, a user selection method which selects statistically orthogonal users for the SE-MAT transmission is proposed to further improve the system performance.
\end{abstract}

\section{INTRODUCTION}

In the downlink, multiuser multiple-input multiple-output (MIMO) technologies can provide high capacity gains by enabling space-division multiple-access (SDMA), but require channel state information (CSI) of all scheduled users at the transmitter [2]-[4]. In frequency division duplexing systems, CSI is obtained at the receiver and fed back to the transmitter [5]. When the feedback delay is larger than the channel coherence time, CSI feedback is outdated and no information about the current channel can be exploited; thus, the conventional multiuser MIMO technologies fail to work.

Recently, Maddah-Ali and Tse proposed a transmission scheme in [1] for such configurations, and their results showed that a degree of freedom (DoF) larger than 1 can be achieved with completely stale CSI at the transmitter (CSIT). This scheme, denoted as MAT, divides the whole transmission period into several phases, and aims at designing the transmit symbols in latter phases using CSI of prior phases. By an ingenious design of the transmitted signal, the objective user can resolve multiple data streams while eliminating all interference. This work has drawn lot of attention in the literature, e.g., see [6]-[9] and the references therein.

In a similar context, we note that compared with instantaneous CSI, statistical CSI (SCSI), such as the knowledge of channel correlation or the channel mean, stays static in a time scale which is much larger than the feedback delay. As a result, it can be obtained at the transmitter easily and accurately. In point-to-point MIMO systems, SCSI-aided transmission schemes have been well studied in the literature, e.g., see [10]-[13] and the references therein. The use of SCSI is also extended to the multiuser scenario in [14], [15]. Inspired by these outdated CSI or SCSI-aided transmission schemes, we make a conjecture that on the basis of the outdated CSI-aided MAT scheme, SCSI can be exploited to further improve the system performance.

On these grounds, we herein propose a downlink transmission scheme, which exploits both SCSI and outdated instantaneous CSI. The proposed scheme extends the MAT scheme by sending symbols demanded by a user along its statistical eigenmodes, instead of directly transmitting through separate antennas, and we refer to it as statistical eigenmode-MAT (SEMAT). Considering zero-forcing (ZF) receiver, we explicitly prove that the proposed SE-MAT scheme can achieve a higher ergodic sum-rate than the original MAT scheme, under different correlation conditions. Moreover, a user selection method which selects statistically orthogonal users for transmission is proposed to further improve the system performance. Our analysis accounts for the effects of spatial correlation on the transmit side, thus is sufficiently general.

The rest of this paper is arranged as follows: The system model is introduced in Section II. In Section III, the SE-MAT scheme is proposed and described in detail. In Section IV, we prove that the proposed SE-MAT scheme can achieve a higher ergodic sum-rate than the original MAT scheme; then, the user selection method is proposed. Numerical results and some discussion are given in Section V.

\section{SySTEM MODEL}

\section{A. Channel Model}

We consider a downlink multiple-input single-output channel where the base station is equipped with $N$ antennas and transmits simultaneously to $N$ users, each of them equipped with one antenna. In time slot $t$, the channel vector between the transmitter and user $k(k=1, \ldots, N)$ is modeled as

$$
\mathbf{h}_{k}(t)=\mathbf{g}_{k}(t)\left(\mathbf{R}_{T, k}^{1 / 2}\right)^{H} \in \mathbb{C}^{1 \times N},
$$

where $\mathbf{g}_{k}(t)$ is a $1 \times N$ vector of independent entries $g_{k, i}(t) \sim$ $\mathcal{C N}(0,1)$ (i.e., zero-mean unit-variance complex Gaussian distributed), the superscript $H$ denotes Hermitian transposition, and $\mathbf{R}_{T, k}^{1 / 2}$ is the square root of the transmit correlation matrix $\mathbf{R}_{T, k}$, which can be decomposed as

$$
\mathbf{R}_{T, k}^{1 / 2}=\mathbf{U}_{T, k} \boldsymbol{\Lambda}_{T, k}^{1 / 2} \mathbf{U}_{T, k}^{H} .
$$


Here, $\mathbf{U}_{T, k}$ is the matrix containing the statistical eigenmodes of user $k$, while

$$
\boldsymbol{\Lambda}_{T, k}^{1 / 2}=\operatorname{diag}\left(\sigma_{k 1}, \ldots, \sigma_{k N}\right),
$$

where $\operatorname{diag}(\cdot)$ denotes the diagonal matrix, with the diagonal entries in the bracket arranged in descending order and normalized such that

$$
\sum_{i=1}^{N} \sigma_{k i}^{2}=N
$$

In addition, we assume that $\mathbf{U}_{T, k}, k=1, \ldots, N$ are uniformly distributed in the unitary space and independent of each other. This assumption can be explained as that the scatterers seen by every user are uniformly and independently distributed in the propagation environment. Referring back to (1), we use $h_{k i}(t)$ to denote the $i$-th element of $\mathbf{h}_{k}(t)$.

In addition, we make the following assumptions:

- The instantaneous CSIT is completely outdated as described in [1], i.e., in time slot $t$, the transmitter only knows $\mathbf{h}_{k}\left(t^{\prime}\right)$ with $t^{\prime}<t$, which is independent of $\mathbf{h}_{k}(t)$.

- Perfect SCSI is available at the transmitter. Recalling from (1), SCSI refers to $\mathbf{R}_{T, k}$, or $\mathbf{U}_{T, k}$ and $\boldsymbol{\Lambda}_{T, k} \cdot{ }^{1}$

\section{B. The Original MAT Scheme}

For the two-user case, the original MAT scheme consists of two phases: Phase 1 contains two slots, and no CSI is used for transmission. Phase 2 contains one slot, and perfect CSI of each user during Phase 1 is exploited in transmission design. The transmitting process is described as follows:

1) Phase 1:

- Slot 1: Transmit $s_{A, i}$ through antenna $i$, for $i=1,2$.

- Slot 2: Transmit $s_{B, i}$ through antenna $i$, for $i=1,2$.

2) Phase 2:

- Slot 3: Transmit $L_{B}(1)+L_{A}(2)$ through antenna 1 . In the above, $s_{k, i}$ denotes the $i$-th data symbol intended for user $k, L_{k}(t)$ denotes the received signal of user $k$ in slot $t$, with $k=A$ or $B$. In slot 3 , a composite signal of order-2 is transmitted, where order- 2 means the signal is useful for both users. In particular, $L_{B}(1)$ is useful for user $A$ and $L_{A}(2)$ is useful for user $B$. With this transmission structure, a DoF of $\frac{4}{3}$ can be achieved by the system.

For the general $N$-user case, the transmitter sends order-1 symbols in Phase 1, designs and sends high-order symbols in the latter phases. The high-order symbols are designed in such a way that the transmitted symbols in former phases can be resolved by the users [1].

\section{Signal Model and ZF Receiver}

Based on the MAT scheme, we can now introduce the signal model for the two-user case. Then, the $\mathrm{ZF}$ receiver, with which the users can resolve their demanded data symbols, is described. In the following, all equations with a superscript

\footnotetext{
${ }^{1}$ SCSI can be either obtained through long-term feedback from the users, or directly estimated at the transmitter using the SCSI reciprocity [16].
}

"MAT" refer to the MAT scheme, while the equations without superscripts can be applied to both schemes, including the MAT scheme and our scheme which will be proposed later.

For the two-user case, the signal vector received by user $A$ over three time slots is written as

$$
\mathbf{y}_{A}=\sqrt{\frac{P}{2}} \overline{\mathbf{H}}_{A 1} \mathbf{s}_{A}+\sqrt{\frac{P}{2}} \overline{\mathbf{H}}_{A 2} \mathbf{s}_{B}+\mathbf{n}_{A},
$$

where $\mathbf{y}_{A}=\left(y_{A}(1), y_{A}(2), y_{A}(3)\right)^{T}$ is the received signal vector with $y_{A}(t)$ denoting the received signal by user $A$ in slot $t$, while $P$ is the total transmit power, $\mathbf{s}_{k}=\left(s_{k, 1}, s_{k, 2}\right)^{T}$ for $k=A, B$, and $\mathbf{n}_{A}$ is the noise vector at user $A$. $\overline{\mathbf{H}}_{A 1}$ and $\overline{\mathbf{H}}_{A 2}$ are two equivalent matrices determined by the transmission scheme. For the original MAT scheme, these are

$$
\begin{aligned}
\overline{\mathbf{H}}_{A 1}^{\mathrm{MAT}} \triangleq\left(\begin{array}{cc}
h_{A 1}(1) & h_{A 2}(1) \\
0 & 0 \\
h_{A 1}(3) h_{B 1}(1) & h_{A 1}(3) h_{B 2}(1)
\end{array}\right) \\
\overline{\mathbf{H}}_{A 2}^{\mathrm{MAT}} \triangleq\left(\begin{array}{cc}
0 & 0 \\
h_{A 1}(2) & h_{A 2}(2) \\
h_{A 1}(3) h_{A 1}(2) & h_{A 1}(3) h_{A 2}(2)
\end{array}\right) .
\end{aligned}
$$

In order to resolve $\mathbf{s}_{A}$ from (5), we use a two-step receiving approach described as follows: In step 1, a linear transformation is made on (5) by left multiplying with a transformation matrix $\mathbf{L}$ to eliminate the interference from the other user:

$$
\mathbf{L y}_{A}=\mathbf{L}\left(\sqrt{\frac{P}{2}} \overline{\mathbf{H}}_{A 1} \mathbf{s}_{A}+\sqrt{\frac{P}{2}} \overline{\mathbf{H}}_{A 2} \mathbf{s}_{B}+\mathbf{n}_{A}\right) .
$$

For the MAT scheme, this transformation matrix reads as

$$
\mathbf{L}^{\mathrm{MAT}} \triangleq\left(\begin{array}{ccc}
1 & 0 & 0 \\
0 & -h_{A 1}(3) & 1
\end{array}\right)
$$

which satisfies

$$
\mathbf{L}^{\mathrm{MAT}} \overline{\mathbf{H}}_{A 2}^{\mathrm{MAT}}=\mathbf{0}_{2 \times 2} .
$$

In step 2, ZF receiver is used in (8) to resolve the two data streams of $s_{A, 1}$ and $s_{A, 2}$. With this approach, the SNRs on the two data streams can be expressed as

$$
\begin{aligned}
\eta_{1}^{\mathrm{MAT}} & =\frac{P}{2} \frac{1}{\left[\left(\tilde{\mathbf{H}}_{A 1}^{\mathrm{MAT}}\right)^{H} \tilde{\mathbf{H}}_{A 1}^{\mathrm{MAT}}\right]_{1,1}^{-1}}, \\
\eta_{2}^{\mathrm{MAT}} & =\frac{P}{2} \frac{1}{2\left[\left(\tilde{\mathbf{H}}_{A 1}^{\mathrm{MAT}}\right)^{H} \tilde{\mathbf{H}}_{A 1}^{\mathrm{MAT}}\right]_{2,2}^{-1}},
\end{aligned}
$$

where $[\mathbf{X}]_{k, k}^{-1}$ denotes the $k$-th diagonal element of $\mathbf{X}^{-1}$, which is the inverse matrix of $\mathbf{X}$, and

$$
\tilde{\mathbf{H}}_{A 1}^{\mathrm{MAT}} \triangleq \mathbf{L}^{\mathrm{MAT}} \overline{\mathbf{H}}_{A 1}^{\mathrm{MAT}} \text {. }
$$

Note that the scaling factor 2 in the denominator in (12) is due to the enhancement of the noise power caused by $\mathbf{L}^{\mathrm{MAT}}$, although this will not affect our analysis in the high-SNR region. The achievable ergodic sum-rate is then expressed as

$$
R_{\mathrm{sum}} \triangleq \sum_{i=1}^{2} \mathbb{E}\left[\log _{2}\left(1+\eta_{i}\right)\right]
$$




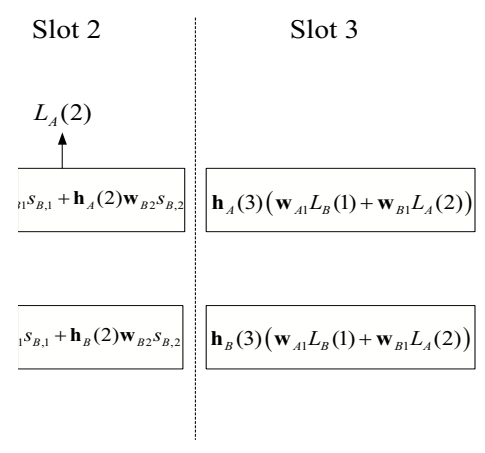

Fig. 1. The SE-MAT system for two users.

\section{THE SE-MAT SCHEME}

In this section, we first outline the proposed SE-MAT scheme for the two-user case, followed by a description of the implementation of this scheme for the general $N$-user case.

\section{A. Two-user Case}

For the two-user case, the proposed SE-MAT scheme is described in Fig. 1 and explained as follows:

1) Phase 1:

- Slot 1: Transmit $s_{A, i}$ along the direction of $\mathbf{w}_{A i}$, for $i=1,2$.

- Slot 2: Transmit $s_{B, i}$ along the direction of $\mathbf{w}_{B i}$, for $i=1,2$.

2) Phase 2 :

- Slot 3: Transmit $L_{B}(1)$ along the direction of $\mathbf{w}_{A 1}$ and $L_{A}(2)$ along the direction of $\mathbf{w}_{B 1}$.

In the above, $\mathbf{w}_{k i}(k=A, B)$ denotes the $i$-th strongest eigen-direction of user $k$, i.e., the $i$-th column vector of $\mathbf{U}_{T, k}$, while $L_{k}(t)$ denotes the received signal of user $k$ in slot $t$, as described in Fig. 1. The main idea for SE-MAT can be explained as follows: In slot 1 , we strengthen $s_{A, 1}$ and $s_{A, 2}$ by applying orthogonally statistical beamforming along the direction of $\mathbf{w}_{A 1}$ and $\mathbf{w}_{A 2}$. In slot 2 , the signals intended for user $B$ are transmitted in a similar manner. In slot 3 , user $A$ needs to know $L_{B}(1)$ to resolve $s_{A, 1}$ and $s_{A, 2}$, while user $B$ needs to know $L_{A}(2)$, thus we send the information demanded by user $A$ or $B$ along the corresponding dominant statistical eigen-direction of each user, respectively.

\section{B. General N-user Case}

For the $N$-user case, the SE-MAT scheme can be generalized as follows:

1) In the transmitting phases, where $N$ order- 1 symbols are transmitted simultaneously to user $k$ in one slot, transmit the $N$ symbols along $N$ statistical eigen-directions of user $k$, instead of transmitting these symbols directly through $N$ transmit antennas, as proposed by the original MAT scheme.

2) In the transmitting phases, where $M$ order- $L$ symbols are transmitted to $L$ users in one slot (The number of $M$ and $L$ is determined by the original MAT scheme as described in [1]), denote the $j$-th order- $L$ symbol which contains information useful for users $1, \ldots, L$ as

$$
I_{(1,2, \ldots, L), j}^{(L)}=\sum_{k=1}^{L} I_{(k), j}^{(1)}, \quad j=1 \ldots M,
$$

where the superscript of $I$ refers to the order of this symbol, and the elements in the bracket in the subscript of $I$ denote the indices of the users for which this information $I$ is useful.

In the original MAT scheme, $M$ symbols described in (15) are directly sent through $M$ separated transmit antennas, while with SE-MAT, we transmit $I_{(k), j}^{(1)}$ along the $j$-th strongest eigen-direction of user $k$.

Note that compared with the MAT scheme, SE-MAT does not change the transmitting structure with respect to phases and slots. The difference between the two schemes is that the latter exploits SCSI to transmit across the statistical eigenmodes, instead of sending symbols directly through separate antennas. Nevertheless, both schemes achieve the same DoF.

\section{RATE ANALYSIS}

In this section, based on the $\mathrm{ZF}$ receiver described in Section II, we show that the ergodic SNR ratio between SE-MAT and the original MAT scheme is larger than 1 on each data stream, and higher ergodic sum-rate can be achieved by SEMAT. Moreover, a user selection method is proposed to further improve the system performance.

We focus on the two-user case. For the SE-MAT scheme, the equivalent channels in (5) can be written as

$$
\begin{aligned}
\overline{\mathbf{H}}_{A 1}^{\mathrm{SE}-\mathrm{MAT}} \triangleq\left(\begin{array}{cc}
\alpha_{A, 1}^{A 1} & \alpha_{A, 1}^{A 2} \\
0 & 0 \\
\alpha_{A, 3}^{A 1} \alpha_{B, 1}^{A 1} & \alpha_{A, 3}^{A 1} \alpha_{B, 1}^{A 2}
\end{array}\right), \\
0 \\
\overline{\mathbf{H}}_{A 1}^{\mathrm{SE}-\mathrm{MAT}} \triangleq\left(\begin{array}{cc}
0 & 0 \\
\alpha_{A, 2}^{B 1} & \alpha_{A, 2}^{B 2} \\
\alpha_{A, 3}^{B 1} \alpha_{A, 2}^{B 1} & \alpha_{A, 3}^{B 1} \alpha_{A, 2}^{B 2}
\end{array}\right),
\end{aligned}
$$

where

$$
\alpha_{k, t}^{l i} \triangleq \mathbf{h}_{k}(t) \mathbf{w}_{l i}
$$

is defined as the inner product between the channel vector $\mathbf{h}_{k}(t)$ and the statistical eigenvector $\mathbf{w}_{l i}$. Note that similar to 
(7), the equivalent interference channel matrix (17) is rank-1, which implies that the interference can be eliminated through a linear transformation of (5). Similar to (9), the transformation matrix can be written as

$$
\mathbf{L}^{\mathrm{SE}-\mathrm{MAT}} \triangleq\left(\begin{array}{ccc}
1 & 0 & 0 \\
0 & -\mathbf{h}_{\mathbf{A}}(3) \mathbf{w}_{\mathbf{B} \mathbf{1}} & 1
\end{array}\right) .
$$

Then, the SNRs on the two data streams reads as

$$
\begin{aligned}
\eta_{1}^{\mathrm{SE}-\mathrm{MAT}} & =\frac{P}{2} \frac{1}{\left[\left(\tilde{\mathbf{H}}_{A 1}^{\mathrm{SE}-\mathrm{MAT}}\right)^{H} \tilde{\mathbf{H}}_{A 1}^{\mathrm{SE}-\mathrm{MAT}]_{1,1}^{-1}},\right.} \\
\eta_{2}^{\mathrm{SE}-\mathrm{MAT}} & =\frac{P}{2} \frac{1}{\left(1+\lambda_{A}^{B 1}\right)\left[\left(\tilde{\mathbf{H}}_{A 1}^{\mathrm{SE}-\mathrm{MAT}}\right)^{H} \tilde{\mathbf{H}}_{A 1}^{\mathrm{SE}-\mathrm{MAT}]_{2,2}^{-1}}\right.},
\end{aligned}
$$

where

$$
\tilde{\mathbf{H}}_{A 1}^{\mathrm{SE}-\mathrm{MAT}} \triangleq \mathbf{L}^{\mathrm{SE}-\mathrm{MAT}} \overline{\mathbf{H}}_{A 1}^{\mathrm{SE}-\mathrm{MAT}}
$$

is the equivalent channel matrix after the transformation, while

$$
\lambda_{l}^{k i} \triangleq \mathbf{w}_{k i}^{H} \mathbf{R}_{T, l} \mathbf{w}_{k i}
$$

is a scaling factor due to the enhancement of the noise power caused by (19). Here, $k=B, i=1$ and $l=A$. Note that according to the Rayleigh-Ritz law for the eigenvalues,

$$
\sigma_{l 2}^{2}<\lambda_{l}^{k i}<\sigma_{l 1}^{2}
$$

\section{A. SNR and Rate Analysis}

We start by comparing $\eta_{i}^{\mathrm{MAT}}$ and $\eta_{i}^{\mathrm{SE}-\mathrm{MAT}}$ for $i=1,2$ to explicitly demonstrate how the SE-MAT scheme increases the average SNR on each data stream. For the sake of simplicity, we consider only the first data stream of user $A$. The proof for the second data stream, as well as the data streams of the other user, can be carried out in a similar manner.

Proposition 1: When the channel is correlated with $\sigma_{A 1}^{2} \geq$ 1.5 , the inequality

$$
\mathbb{E}\left[\frac{\eta_{1}^{\mathrm{SE}-\mathrm{MAT}}}{\eta_{1}^{\mathrm{MAT}}}\right]>1
$$

strictly holds.

Proof: See Appendix I.

We denote the left hand side of (25) as the ergodic SNR ratio. The condition $\sigma_{A 1}^{2}>1.5$ corresponds to a certain channel correlation level. In practice, we can adjust the interantenna spacing to satisfy this condition, as smaller interantenna spacing will result in a larger value of $\sigma_{A 1}^{2}$. However, a very interesting case is when all users are assumed to have the same $\boldsymbol{\Lambda}_{k}$ in (2). This assumption is sufficiently realistic when all users are located in the same scattering environment and share the same correlation level [18]. With this assumption, (25) can be proved without any restriction on the value of $\sigma_{A 1}^{2}$. The results are described in the following corollary.

Corollary 1: When all users share the same correlation profile such that

$$
\boldsymbol{\Lambda}_{T, k}^{1 / 2}=\boldsymbol{\Lambda}_{T}^{1 / 2}, k=1, \ldots, N,
$$

(25) strictly holds for any value of $\sigma_{A 1}^{2}$ and $\sigma_{B 1}^{2}$.

Proof: The proof can be done in a similar way as that used in proving Proposition 1, and is omitted here.

Further, we extend the analysis to the achievable ergodic rate in the following proposition.

Proposition 2: In the high SNR region, the proposed SEMAT scheme achieves a higher ergodic sum-rate than the original MAT scheme, with the channel correlation profile stated in Proposition 1 or Corollary 1.

Proof: See Appendix II.

Remark 1: Although the proof relies on a high-SNR assumption, it is shown in Section V that SE-MAT can achieve a higher ergodic sum-rate, than the original MAT scheme, at arbitrary SNRs as well as arbitrary channel correlation levels.

\section{B. SE-MAT with User Selection}

From (38) in the proof of Proposition 1, we can infer that in order to maximize the lower bound $f\left(\lambda_{B}^{A 2}\right)$ of the ergodic SNR ratio, $\lambda_{B}^{A 2}$ is required to be as large as possible. To achieve the maximum value $\sigma_{B 1}^{2}$, it is required that

$$
\mathbf{w}_{A 2}=\mathbf{w}_{B 1},
$$

which indicates that the two users need to have statistically orthogonal eigenmodes. In practice, a codebook-based user grouping algorithm can be used to select users in such a way. A detailed description of such an algorithm is beyond the scope of this paper, and interested readers are referred to [15].

With user selection, the ergodic SNR ratio between SEMAT and MAT is enhanced. Although we can not conclude that the ergodic data rate is also improved, simulation results in Section $\mathrm{V}$ will show that significant rate gains can be obtained compared to the schemes without user selection.

\section{NuMERICAL RESUltS AND DiscUSSION}

In this section, numerical results are shown for the two-user case. The ergodic sum-rates are calculated using (14), where the expectation is taken by averaging over 100 snapshots, with 3000 time slots in every snapshot. Here, one snapshot means a random generation of $\mathbf{U}_{T, k}$ in (2). In every time slot, $g_{k}(t)$ in (1) is independently and identically generated. For convenience of description, we set $\sigma_{k 1}^{2}=\sigma_{1}^{2}$ and $\sigma_{k 2}^{2}=\sigma_{2}^{2}$ ( $k=A, B)$ in (3), and assume these values are fixed over all snapshots. At last, the achievable rates are divided by the number of the total time slots used for one transmission.

In Fig. 2, the achievable ergodic sum-rates per time slot are illustrated. We set $\sigma_{1}^{2}=1.8$ and $\sigma_{2}^{2}=0.2$ as an example. In the figure, "US" stands for "user selection" which means the two users are selected to satisfy (27). The figure shows that by strengthening the desired signal on the statistical eigenmodes of the objective user, SE-MAT achieves a higher sum-rate compared to the original MAT scheme, while keeping the same DoF, which is indicated by the slope of the curves. When the two users are selected to be statistically orthogonal, the achievable ergodic sum-rate can be further improved.

In Fig. 3, the ergodic sum-rate ratios $R_{\mathrm{sum}}^{\mathrm{SE}-\mathrm{MAT}} / R_{\mathrm{sum}}^{\mathrm{MAT}}$ are depicted versus the value of $\chi$, which is defined as $\chi=\frac{\sigma_{1}^{2}}{\sigma_{2}^{2}}$, 


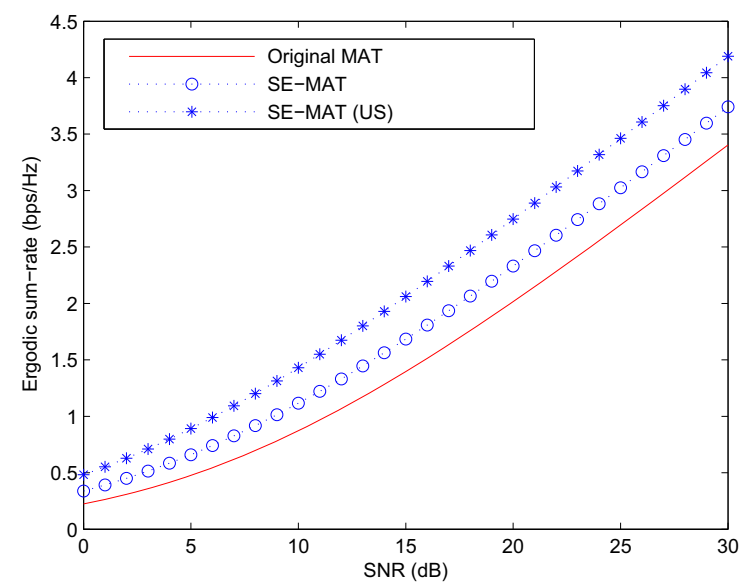

Fig. 2. Ergodic sum-rate per time slot vs. SNR.

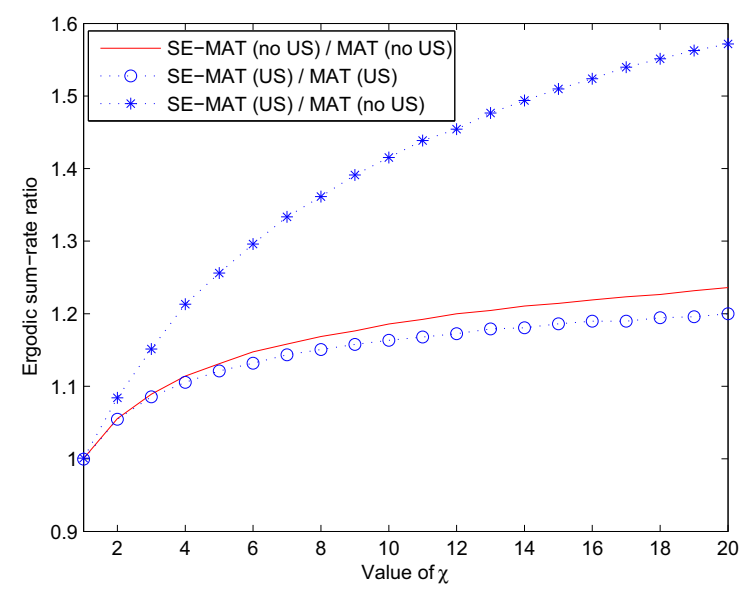

Fig. 3. Ergodic sum-rate ratio vs. $\chi$ at $\mathrm{SNR}=20 \mathrm{~dB}$.

and indicates the channel correlation level. In particular, we compare the ergodic sum-rate ratios for three cases, these are:

- The rate ratio between SE-MAT and the original MAT, when both schemes are used without user selection

- The rate ratio between SE-MAT and the original MAT, when both schemes are combined with user selection

- The rate ratio between SE-MAT with user selection, and the original MAT without user selection.

Fig. 3 shows that the rate ratio between the two schemes increases with the value of $\chi$. Considering user selection for both schemes, the rate ratio becomes lower than that achieved without user selection. This is because selecting statistically orthogonal users also plays a positive role in the original MAT scheme. However, it is shown that combining user selection and SE-MAT can provide a dramatic gain in the achievable rate, when compared with the original MAT scheme without user selection. These gains are more pronounced with higher spatial correlation, i.e., larger values of $\chi$.

\section{CONCLUSION}

On basis of the MAT scheme which exploits outdated CSIT in multiuser downlink transmission, we further made use of statistical CSIT and proposed a scheme named as SE-MAT exploiting both kinds of CSI. This scheme transmits through the statistical eigenmodes of each user, instead of transmitting directly through antennas. Theoretical analysis and numerical results showed that a higher sum-rate can be achieved by SEMAT compared with the original MAT scheme, and the rate improvement increases as the channel correlation increases.

\section{APPENDIX I PROOF OF PROPOSITION 1}

For the first stream, we get

$$
\frac{\eta_{1}^{\mathrm{SE}-\mathrm{MAT}}}{\eta_{1}^{\mathrm{MAT}}}=\frac{\left[\mathbf{M}^{\mathrm{MAT}}\right]_{1,1}^{-1}}{\left[\mathbf{M}^{\mathrm{SE}-\mathrm{MAT}}\right]_{1,1}^{-1}}
$$

where $\mathbf{M}=\tilde{\mathbf{H}}_{A 1}^{H} \tilde{\mathbf{H}}_{A 1}$ (With the superscript being either "MAT" or "SE-MAT"). For MAT, M MAT can be written as (29) (See bottom of this page). For SE-MAT, $\mathbf{M}^{\mathrm{SE}-\mathrm{MAT}}$ reads as (30) (See bottom of this page). Noting that

$$
[\mathbf{X}]_{k, k}^{-1}=\frac{\operatorname{det}\left(\mathbf{X}_{k, k}\right)}{\operatorname{det}(\mathbf{X})}
$$

where $\mathbf{X}_{k, k}$ denotes the minor matrix of $\mathbf{X}$, i.e., the submatrix obtained by omitting the $k$-th row and the $k$-th column of $\mathbf{X}$, (28) can be written as

$$
\frac{\eta_{1}^{\mathrm{SE}-\mathrm{MAT}}}{\eta_{1}^{\mathrm{MAT}}}=\frac{\operatorname{det}\left(\mathbf{M}^{\mathrm{SE}-\mathrm{MAT}}\right) \operatorname{det}\left(\mathbf{M}_{1,1}^{\mathrm{MAT}}\right)}{\operatorname{det}\left(\mathbf{M}^{\mathrm{MAT}}\right) \operatorname{det}\left(\mathbf{M}_{1,1}^{\mathrm{SE}-\mathrm{MAT}}\right)} .
$$

Taking expectation at both sides of (32), and using Mullen's inequality ${ }^{2}$, we end up with

$$
\mathbb{E}\left[\frac{\eta_{1}^{\mathrm{SE}-\mathrm{MAT}}}{\eta_{1}^{\mathrm{MAT}}}\right] \geqslant \frac{\mathbb{E}\left[\operatorname{det}\left(\mathbf{M}^{\mathrm{SE}-\mathrm{MAT}}\right)\right] \mathbb{E}\left[\operatorname{det}\left(\mathbf{M}_{1,1}^{\mathrm{MAT}}\right)\right]}{\mathbb{E}\left[\operatorname{det}\left(\mathbf{M}^{\mathrm{MAT}}\right)\right] \mathbb{E}\left[\operatorname{det}\left(\mathbf{M}_{1,1}^{\mathrm{SE}-\mathrm{MAT}}\right)\right]},
$$

${ }^{2}$ The Mullen's inequality reads as $\mathbb{E}\left[\frac{X}{Y}\right] \geq \frac{\mathbb{E}[X]}{\mathbb{E}[Y]}$, where $X$ and $Y$ are independent random variables [17].

$$
\mathbf{M}^{\mathrm{MAT}}=\left(\begin{array}{cc}
\left|h_{A 1}(1)\right|^{2}+\left|h_{A 1}(3)\right|^{2}\left|h_{B 1}(1)\right|^{2} & h_{A 1}^{*}(1) h_{A 2}(1)+\left|h_{A 1}(3)\right|^{2} h_{B 1}^{*}(1) h_{B 2}(1) \\
h_{A 1}(1) h_{A 2}^{*}(1)+\left|h_{A 1}(3)\right|^{2} h_{B 1}(1) h_{B 2}^{*}(1) & \left|h_{A 2}(1)\right|^{2}+\left|h_{A 1}(3)\right|^{2}\left|h_{B 2}(1)\right|^{2}
\end{array}\right) .
$$

$$
\mathbf{M}^{\mathrm{SE}-\mathrm{MAT}}=\left(\begin{array}{cc}
\left|\alpha_{A, 1}^{A 1}\right|^{2}+\left|\alpha_{A, 3}^{A 1}\right|^{2}\left|\alpha_{B, 1}^{A 1}\right|^{2} & \left(\alpha_{A, 1}^{A 1}\right)^{*} \alpha_{A, 1}^{A 2}+\left|\alpha_{A, 3}^{A 1}\right|^{2}\left(\alpha_{B, 1}^{A 1}\right)^{*} \alpha_{B, 1}^{A 2} \\
\alpha_{A, 1}^{A 1}\left(\alpha_{A, 1}^{A 2}\right)^{*}+\left|\alpha_{A, 3}^{A 1}\right|^{2} \alpha_{B, 1}^{A 1}\left(\alpha_{B, 1}^{A 2}\right)^{*} & \left|\alpha_{A, 1}^{A 2}\right|^{2}+\left|\alpha_{A, 3}^{A 1}\right|^{2}\left|\alpha_{B, 1}^{A 2}\right|^{2}
\end{array}\right) .
$$


where it can be calculated that

$$
\begin{aligned}
\mathbb{E}\left[\operatorname{det}\left(\mathbf{M}_{1,1}^{\mathrm{MAT}}\right)\right] & =2, \\
\mathbb{E}\left[\operatorname{det}\left(\mathbf{M}_{1,1}^{\mathrm{SE}-\mathrm{MAT}}\right)\right] & =\sigma_{A 2}^{2}+\sigma_{A 1}^{2} \lambda_{B}^{A 2},
\end{aligned}
$$

where $\lambda_{l}^{k i}$ is defined in (23), while

$$
\begin{aligned}
\mathbb{E}\left[\operatorname{det}\left(\mathbf{M}^{\mathrm{MAT}}\right)\right] & =2, \\
\mathbb{E}\left[\operatorname{det}\left(\mathbf{M}^{\mathrm{SE}-\mathrm{MAT}}\right)\right] & =\sigma_{A 1}^{2}\left(\sigma_{A 1}^{2} \lambda_{B}^{A 2}+\sigma_{A 2}^{2} \lambda_{B}^{A 1}\right) .
\end{aligned}
$$

Substituting (34)-(37) into (33), we get

$$
\begin{aligned}
\mathbb{E}\left[\frac{\eta_{1}^{\mathrm{SE}-\mathrm{MAT}}}{\eta_{1}^{\mathrm{MAT}}}\right] & \geqslant \frac{\sigma_{A 1}^{2}\left(\sigma_{A 1}^{2} \lambda_{B}^{A 2}+\sigma_{A 2}^{2} \lambda_{B}^{A 1}\right)}{\sigma_{A 2}^{2}+\sigma_{A 1}^{2} \lambda_{B}^{A 2}} \\
& =\frac{\sigma_{A 1}^{2}\left(\sigma_{A 1}^{2} \lambda_{B}^{A 2}+\left(2-\sigma_{A 1}^{2}\right)\left(2-\lambda_{B}^{A 2}\right)\right)}{\sigma_{A 2}^{2}+\sigma_{A 1}^{2} \lambda_{B}^{A 2}} \\
& \triangleq f\left(\lambda_{B}^{A 2}\right) .
\end{aligned}
$$

The equality in (38) holds for the reason that

$$
\begin{gathered}
\lambda_{B}^{A 1}+\lambda_{B}^{A 2}=2, \\
\sigma_{A 1}^{2}+\sigma_{A 2}^{2}=2
\end{gathered}
$$

according to (4). It is easy to show that $f\left(\lambda_{B}^{A 2}\right)$ in (38) is a decreasing function with respect to $\lambda_{B}^{A 2}$, thus the minimum value of (38) is achieved when $\lambda_{B}^{A 2}$ achieves its maximum value, which is $\sigma_{B 1}^{2}$ according to (24). As a result,

$$
f\left(\lambda_{B}^{A 2}\right) \geq f\left(\sigma_{B 1}^{2}\right)=\frac{\sigma_{A 1}^{2}\left(\sigma_{A 1}^{2} \sigma_{B 1}^{2}+\left(2-\sigma_{A 1}^{2}\right)\left(2-\sigma_{B 1}^{2}\right)\right)}{2-\sigma_{A 1}^{2}+\sigma_{A 1}^{2} \sigma_{B 1}^{2}} .
$$

Note that (41) is increasing with respect to $\sigma_{A 1}^{2}$. As a result, when $\sigma_{A 1}^{2}>1.5$,

$$
f\left(\lambda_{B}^{A 2}\right) \geq\left. f\left(\sigma_{B 1}^{2}\right)\right|_{\sigma_{A 1}^{2}=1.5}=\frac{1.5\left(\sigma_{B 1}^{2}+1\right)}{0.5+1.5 \sigma_{B 1}^{2}}>1 .
$$

Recalling (38), the proposition can be proved.

\section{APPENDIX II}

\section{ProOF OF PROPOSITION 2}

At high SNRs, the rate gap on the first data stream between the two schemes can be written as

$$
\begin{aligned}
\Delta R & =\mathbb{E}\left[\log _{2} \eta_{1}^{\mathrm{SE}-\mathrm{MAT}}\right]-\mathbb{E}\left[\log _{2} \eta_{1}^{\mathrm{MAT}}\right] \\
& =\mathbb{E}\left[\log _{2} \frac{\eta_{1}^{\mathrm{SE}-\mathrm{MAT}}}{\eta_{1}^{\mathrm{MAT}}}\right] .
\end{aligned}
$$

Using the inequality

$$
\log (x)>\frac{x-1}{x}, \text { for } x>0,
$$

(43) can be bounded as

$$
\Delta R>\mathbb{E}\left[\frac{\kappa-1}{\kappa}\right]
$$

where $\kappa \triangleq \eta_{1}^{\mathrm{SE}-\mathrm{MAT}} / \eta_{1}^{\mathrm{MAT}}$. Using Mullen's inequality once more, (45) can be further bounded as

$$
\Delta R>\frac{\mathbb{E}[\kappa-1]}{\mathbb{E}[\kappa]} \text {. }
$$

Recalling from Proposition 1 and Corollary 1 that $\mathbb{E}[\kappa]>1$ in correlated channels with the stated correlation profile, we get that $\Delta R>0$, which completes the proof.

\section{ACKNOWLEDGMENT}

This work was supported by the National Natural Science Foundation of China under Grants 61222102 and 60902009, the Natural Science Foundation of Jiangsu Province under Grants BK2012021, and the Supporting Program for New Century Excellent Talents in University (NCET-11-0090). The work of M. Matthaiou has been supported in part by the Swedish Governmental Agency for Innovation Systems (VINNOVA) within the VINN Excellence Center Chase.

\section{REFERENCES}

[1] M. A. Maddah-Ali and D. Tse, "Completely stale transmitter channel state information is still very useful," preprint, 2011. [Online] Available at http://arxiv.org/abs/1010.1499.

[2] N. Jindal and A. Goldsmith, "Dirty paper coding vs. TDMA for MIMO broadcast channels," IEEE Trans. Inf. Theroy, vol. 51, no. 5, pp. 1783 1794, May 2005.

[3] K.-K. Wong, R. D. Murch, and K. B. Letaief, "A joint-channel diagonalization for multiuser MIMO antenna systems," IEEE Trans. Wireless Commun., vol. 4, no. 2, pp. 773-786, July 2003.

[4] Z. G. Pan, K.-K. Wong, and T. S. Ng, "Generalized multiuser orthogonal space division multiplexing," IEEE Trans. Wireless Commun., vol. 3, no. 6, pp. 1969-1973, Nov. 2004.

[5] D. J. Love, R. W. Heath, Jr., V. K. N. Lau, D. Gesbert, B. D. Rao, and M. Andrews, "An overview of limited feedback in wireless communication systems," IEEE J. Sel. Areas Commun., vol. 26, no. 8, pp. 1341-1365, Oct. 2008

[6] H. Maleki, S. A. Jafar, and S. Shamai (Shitz), "Retrospective interference alignment," preprint, 2011. [Online] Available at http://arxiv.org/abs/1009.3593.

[7] A. Ghasemi, A. S. Motahari, and A. K. Khandani, "On the degrees of freedom of X channel with delayed CSIT," in Proc. IEEE Int. Symp. Inf. Theory (ISIT), July 2011, pp. 909-912.

[8] C. S. Vaze and M. K. Varanasi, "The degrees of freedom region of the two-user MIMO broadcast channel with delayed CSI," preprint, 2011. [Online] Available at http://arxiv.org/abs/1101.0306.

[9] J. Xu, J. G. Andrews, and S. A. Jafar, "Broadcast channels with delayed finite-rate feedback: Predict or observe?," preprint, 2011. [Online] Available at http://arxiv.org/abs/1105.3686.

[10] V. Veeravalli, Y. Liang, and A. M. Sayeed, "Correlated MIMO wireless channels: Capacity, optimal signaling and asymptotics," IEEE Trans. Inf. Theory, vol. 51, no. 6, pp. 2058-2072, June 2005.

[11] S. A. Jafar and A. J. Goldsmith, "Transmitter optimization and optimality of beamforming for multiple antenna systems," IEEE Trans. Wireless Commun., vol. 3, no. 4, pp. 1165-1175, July 2004.

[12] A. M. Tulino, A. Lozano, and S. Verdú, "Capacity-achieving input covariance for single-user multi-antenna channels," IEEE Trans. Wireless Commun., vol. 5, no. 3, pp. 662-671, Mar. 2006.

[13] X. Q. Gao, B. Jiang, X. Li, A. B. Gershman, and M. R. McKay, "Statistical eigenmode transmission over jointly-correlated MIMO channels," IEEE Trans. Inf. Theory, vol. 55, no. 8, pp. 3735-3750, Aug. 2009.

[14] V. Raghavan, S. V. Hanly, and V. V. Veeravalli, "Statistical beamforming on the Grassmann manifold for the two-user broadcast channel," preprint, 2011. [Online] Available at http://arxiv.org/abs/1104.2116.

[15] J. Wang, S. Jin, X. Q. Gao, K.-K. Wong and E. Au, "Statistical eigenmode-based SDMA for two-user downlink," submitted to IEEE Trans. Signal Process., 2011.

[16] B. K. Chalise, L. Haering, and A. Czylwik, "Robust uplink to downlink spatial covariance matrix transformation for downlink beamforming," in Proc. IEEE Int. Conf. Commun. (ICC), June 2004, vol. 5, pp. 30103014.

[17] K. Mullen, "A note on the ratio of two independent random variables," The American Statisticians, vol. 21, no. 3, pp. 30-31, Jun. 1967.

[18] B. Clerckx, G. Kim, and S. Kim, "MU-MIMO with channel statisticsbased codebooks in spatially correlated channels," in Proc. IEEE Global Telecommun. Conf. (GLOBECOM), Dec. 2008, pp. 956-960. 